\title{
Through the dark continent: African trypanosome development in the tsetse fly
}

\author{
Brice Rotureau $^{1 *}$ and Jan Van Den Abbeele ${ }^{2,3}$ \\ 1 Trypanosome Cell Biology Unit, Institut Pasteur and CNRS URA 2581, Paris, France \\ 2 Unit of Veterinary Protozoology, Department of Biomedical Sciences, Institute of Tropical Medicine, Antwerp, Belgium \\ ${ }^{3}$ Laboratory of Zoophysiology, Department of Physiology, University of Ghent, Ghent, Belgium
}

\section{Edited by:}

Charles L. Jaffe, Hebrew

University-Hadassah Medical

School, Israel

\section{Reviewed by:}

Hua Xie, Meharry Medical College, USA

Ashu Sharma, University at Buffalo, State University of New York, USA

Serap Aksoy, Yale University, USA

\section{*Correspondence:}

Brice Rotureau, Trypanosome Cell

Biology Unit, Institut Pasteur and

CNRS USA 2581, 25 rue du Docteur

Roux, 75015 Paris, France

e-mail: rotureau@pasteur.fr
African trypanosomes are unicellular flagellated parasites causing trypanosomiases in Africa, a group of severe diseases also known as sleeping sickness in human and nagana in cattle. These parasites are almost exclusively transmitted by the bite of the tsetse fly. In this review, we describe and compare the three developmental programs of the main trypanosome species impacting human and animal health, with focus on the most recent observations. From here, some reflections are made on research issues concerning trypanosome developmental biology in the tsetse fly that are to be addressed in the future.

Keywords: African trypanosomes, development, parasite cycle, tsetse fly, vector

\section{INTRODUCTION}

African trypanosomiases are a set of vector-borne diseases of humans and their livestock, which have devastating socio-economic consequences for the Sub-Saharan African continent. They result from infections with flagellated unicellular parasites named African trypanosomes (Kinetoplastida: Trypanosomatidae) of which the majority is exclusively transmitted by the bite of tsetse flies (Diptera: Glossinidae). Two species of African trypanosomes are responsible for Human African Trypanosomiasis (HAT), also known as sleeping sickness, whereas at least seven other species cause Animal African Trypanosomiasis (AAT) or nagana (Table 1).

Trypanosoma brucei rhodesiense and T. b. gambiense are the causative agents of HAT in East/Southern Africa and West/Central Africa, respectively. The T. $b$. rhodesiense transmission cycle mainly involves wild and domestic animals, but intensified human-to-human transmission may occur during epidemics. The T. b. gambiense transmission cycle is mostly from human to human with occasional involvement of an animal reservoir. There are no prophylactic drugs or vaccines available for HAT and the few available treatments present a complex posology and severe side effects (Fevre et al., 2006; Brun et al., 2009). It is estimated that $\sim 70$ million people living in tsetse fly-infested areas are at different levels of risk of contracting HAT, especially in countries such as the Democratic Republic of Congo (DRC), Angola, South-Sudan, and the Central African Republic (Simarro et al., 2008, 2010, 2012; WHO, 2012). In 2011, less than 10,000 new cases were reported (Simarro et al., 2012), but probably more cases remained undetected given that sleeping sickness occurs in remote rural areas.
While these African trypanosome species are important for public health, other species cause severe disease in livestock (Table 1). T. vivax and T. congolense are the major pathogens of cattle and other ruminants, while T. simiae, T. godfreyi, and T. suis cause high mortality in domestic pigs. AAT restricts agricultural development on the African continent despite the availability of prophylactic and curative drugs. Moreover, it is worrying to see drug effectiveness being seriously threatened by an increasing drug resistance in animal trypanosomes (Delespaux et al., 2008).

In contrast to sand flies and mosquitoes, both male and female tsetse flies are obligatory blood feeders and are able to transmit trypanosomes. All pathogenic African trypanosomes are called Salivarian as they are transmitted via the saliva during the fly feeding. Tsetse flies are the exclusive cyclical insect vectors and it can be assumed that all species of Glossina could act as vectors (Table 1). Therefore, vector-oriented control is one of the main pillars in the fight against HAT and AAT to reduce parasite transmission and dissemination. In addition, direct mechanical transmission by other haematophagous flies such as tabanids and Stomoxys frequently occurs for T. vivax.

A comprehensive understanding of the trypanosome developmental pathway in the tsetse fly and the interactions that affect this journey is of high importance that will allow a better understanding of the transmission dynamics of these parasites in the natural context and the improvement of current transmission control measures. Recent reviews already present in-depth overviews of our knowledge on the tsetse-trypanosome interactions (Aksoy et al., 2003; Roditi and Lehane, 2008; Walshe et al., 2009), especially for T. $b$. brucei (Sharma et al., 2009; Dyer et al., 2013). Additionally, recent experimental work demonstrated the 


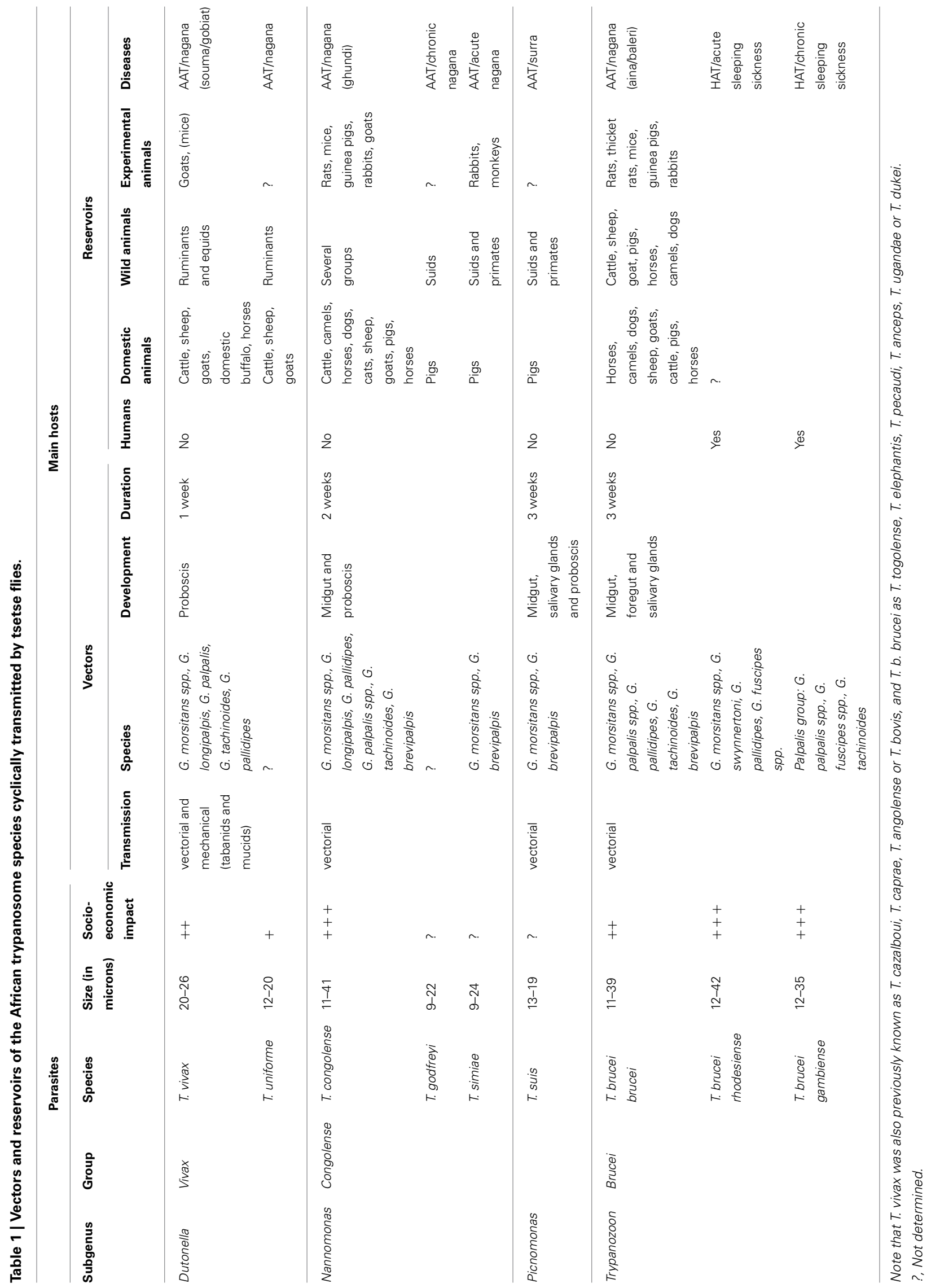


importance of microbiome-associated tsetse fly immunity in trypanosome development (Weiss et al., 2012, 2013). In this minireview, we will focus on the major advancements during the past 5 years in our understanding of the trypanosome developmental programs inside the tsetse fly. We will compare the life cycles of the three epidemiologically most relevant trypanosome species, namely $T$. vivax, T. congolense, and T. brucei.

\section{AFRICAN TRYPANOSOME DEVELOPMENTAL CYCLES IN THE TSETSE FLY}

A tsetse fly picks up trypanosomes during the acquisition of a blood meal on an infected mammal. Within the fly, the parasites have to go through a developmental cycle that can be simple or complex depending on the trypanosome species, consisting of several steps of proliferation, migration and differentiation. The final goal is to differentiate into infective metacyclic trypanosomes that are ready for transmission to the next mammalian host. The completion of this developmental cycle may take from a few days for T. vivax up to 3 weeks for T. brucei. It requires specific adaptations of the parasite to the different tsetse fly microenvironments, involving metabolic, cell surface protein or striking morphological modifications. Three distinct developmental programs have been described among Salivarian trypanosomes according to the complexity of their journey in the tsetse alimentary tract (Table 1 and Figure 1). Development of parasites of the $T$. vivax group (subgenus Duttonella) is restricted to the tsetse proboscis and cibarium. These parasites are believed to be the most ancient of the Salivarian trypanosomes.

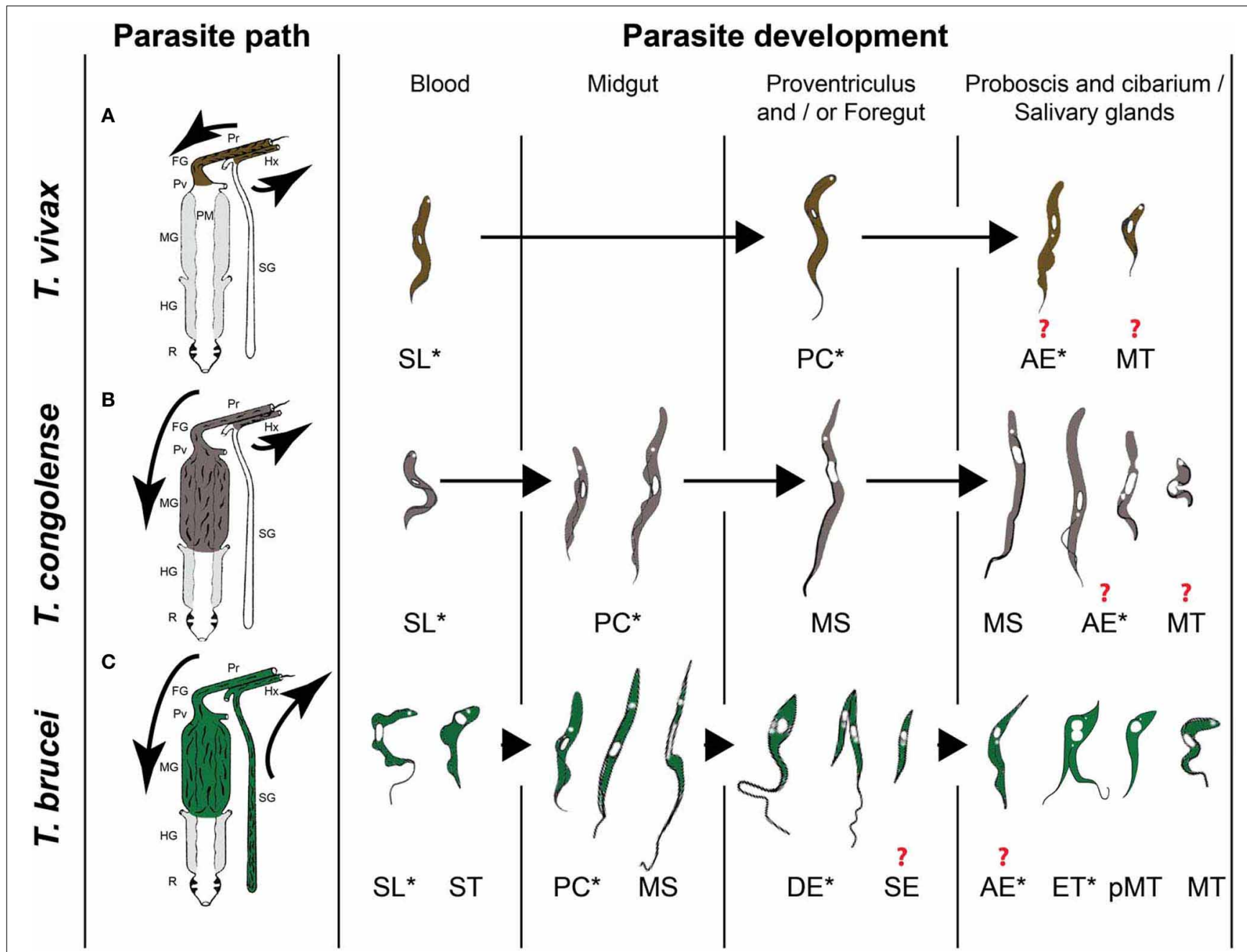

FIGURE 1 | The three types of African trypanosome development in the tsetse fly. (A) T. vivax group. (B) T. congolense group. (C) T. brucei group. Parasite paths in the tsetse digestive tract are schematically presented in the left panel [adapted from (Hoare, 1972)]. Successive parasite stages found in the different organs are presented in a chronological order in the right panel [adapted from (Hoare, 1972;

Peacock et al., 2007, 2012; Rotureau et al., 2012)]. * indicate proliferating stages and ? indicate an uncertainty with respect to the type of division and/or the transitional forms involved at this stage of development. Pr: proboscis, FG: foregut, $\mathrm{PV}$ : proventriculus, $\mathrm{PM}$ : peritrophic matrix, MG: midgut, HG: hindgut, $\mathrm{R}$ : rectum, $\mathrm{Hx}$ : hypopharynx, SG: salivary glands, SL: slender trypomastigote, ST: stumpy trypomastigote, PC: procyclic trypomastigote, MS: mesocyclic trypomastigote, DE: long dividing epimastigote, SE: short epimastigote, AE: attached epimastigote, ET: epi-trypo dividing epimastigote, pMT: pre-metacyclic trypomastigote, MT: metacyclic trypomastigote. 
Trypanosomes of the Nannomonas subgenus comprise three species (including the economically important $T$. congolense) that successively develop in the midgut, foregut and proboscis of the flies. The T. brucei group (Trypanozoon subgenus) contains three trypanosome species (including the human-pathogenic T. b. gambiense and T. $b$. rhodesiense) that successively develop in the tsetse midgut, foregut, proboscis, and salivary glands. A remarkable common feature that occurs in three developmental programs is the switch between two morphotypes, i.e., the trypomastigote and epimastigote morphotype. These morphotypes are defined according to the relative position of the kinetoplast (condensed mitochondrial DNA) to the nucleus (Hoare and Wallace, 1966). In trypomastigotes, the kinetoplast localizes between the nucleus and the posterior end of the cell, whereas in epimastigote forms it is positioned at the other side, i.e., between the nucleus and the anterior end of the cell. This morphotype switch implies a drastic internal re-organization of the nucleus and kinetoplast that is assumed to be a costly cellular event. Therefore, the occurrence of this switch in all these cyclical developmental programs suggests that it plays an essential role in the parasite life cycle. The further very distinct developmental pathways of the three different trypanosome groups could be the result of a long-term co-evolution between parasites and their vectors that minimizes inter-trypanosome competition within the tsetse fly in order to maximize their respective transmission.

\section{PART I: THE EPIMASTIGOTE'S RISE}

In $T$. vivax and $T$. congolense, a single type of trypomastigote is found to proliferate in the bloodstream of the mammalian host. In contrast, two distinct types of $T$. brucei bloodstream parasites are distinguished: the dividing slender trypomastigote and the non-dividing tsetse-infective stumpy trypomastigote that is observed at peaks of parasitemia. Few hours after ingestion, T. brucei and T. congolense bloodstream trypomastigotes differentiate into procyclic trypomastigotes in the posterior midgut of the fly where they start multiplication (Figure 1). A number of these procyclics cross the peritrophic matrix, progressively elongate and migrate to the anterior part of the midgut as non-proliferative long mesocyclic trypomastigotes (Figure 1). Once in the proventriculus (cardia), T. brucei mesocyclic cells become thinner and adopt an epimastigote configuration accompanied by the migration of the nucleus to the posterior side of the kinetoplast (Sharma et al., 2008). This phenomenon was perturbed upon forced expression of the stress granule-associated protein ALBA3, showing for the first time the involvement of an RNA-binding protein in trypanosome development (Subota et al., 2011). Next, the long "tadpole-like" or "spermatozoa-like" epimastigotes start to divide asymmetrically in the proventriculus and foregut to produce a long and a short epimastigote (Van Den Abbeele et al., 1999) (Figure 1C). This dividing stage does not display any increase in cell volume neither in cell length, but is marked by a profound remodeling of the posterior part of the cytoskeleton and by changes in the molecular composition and/or organization of the flagellum attachment zone (Rotureau et al., 2011). In contrast to $T$. brucei, long $T$. congolense trypomastigotes (similar to the T. brucei mesocyclic forms) stop division in the proventriculus and become uniform in size. They retain a trypomastigote morphology during migration via the foregut to the proboscis where the trypomastigote-epimastigote transition takes place without any evident asymmetric division (Peacock et al., 2012) (Figure 1B). For T. vivax, only a small number of the ingested bloodstream forms remains in the foregut and cibarial region and undergoes a short developmental cycle from the trypomastigote into the epimastigote form (Moloo and Gray, 1989) (Figure 1A).

\section{PART II: THE TRYPOMASTIGOTE'S REVENGE}

For T. congolense, the long trypomastigotes in the foregut lumen migrate to the cibarium and proboscis and become epimastigotes that attach to the chitinous lining (rosette formation) where they proliferate and develop into infective metacyclics (Figure 1B). Dividing trypomastigotes and epimastigotes, as well as parasites in transition between the two morphotypes were observed at the same time in the proboscis (Peacock et al., 2012). Therefore, it remains uncertain whether these morphological transitions are mediated by cell division or by a differentiation event. T. brucei moves upstream from the proventriculus to the salivary glands via the foregut and proboscis while undergoing a first asymmetric division. Here, it is assumed that the long and highly motile epimastigote form acts as a transport vehicle that generates the short epimastigote in the salivary gland lumen by completing the asymmetric division (Figure 1C). Whether this process occurs continuously or only in a narrow time frame after parasites have reached the foregut is still under debate (Van Den Abbeele et al., 1999). However, based upon experimental work with tagged parasites, it is clear that only very few parasites (0-5) are successful in completing this migration process (Oberle et al., 2010).

Once in the salivary glands, the short epimastigote parasites attach to the epithelium via their flagellum and elongate (Tetley and Vickerman, 1985; Sharma et al., 2009) (Figure 1C). Recently, two distinct cycles of trypanosome proliferation were demonstrated to occur simultaneously in the salivary glands (Rotureau et al., 2012). The first cycle produces two equivalent cells that are attached to the epithelium. This mode of proliferation is predominant during the early phase of infection and ensures a rapid colonization of the glands. The second cycle is more frequent at a later stage and involves an asymmetric division (Figure 1C). It produces a daughter cell that matures into the infective metacyclic form that is released in the saliva, as demonstrated by the expression of specific molecular markers. It has been proposed that the coordination of these two alternative cell cycles contributes to the continuous production of infective parasites (Rotureau et al., 2012). In addition, T. brucei metacyclogenesis was shown to induce a severe modification of the tsetse salivary composition resulting in a drastically reduced anti-haemostatic potential and a hampered feeding performance, which could lead to an increased vector/host contact and parasite transmission in field conditions (Van Den Abbeele et al., 2010).

Although T. vivax development appears to be more simple, it remains poorly studied. Trypomastigote and epimastigote parasites from the foregut and cibarium migrate to the proboscis. Subsequent invasion of the hypopharynx by some of these forms leads to the further transformation into the infective metacyclic 
forms (Jefferies et al., 1987; Moloo and Gray, 1989). As for T. congolense, the exact developmental program from the trypomastigote to the epimastigote, and back to the trypomastigote morphotype remains to be unravelled: does it involve an asymmetric division as described for T. brucei or does it depend on a differentiation event?

It is clear that both T. brucei and T. congolense parasites go through a complex and tortuous developmental program in the tsetse fly vector. This strategy confers important advantages to the parasite such as multiple transmission opportunities to new mammalian hosts during the entire life of the tsetse fly as well as the opportunity for genetic (sexual) exchange (Aksoy et al., 2003).

\section{SEX AND THE EPI}

T. brucei experiences a pronounced bottleneck during differentiation and migration from the midgut to the salivary glands (Oberle et al., 2010). An important outcome of this bottleneck is that rare variants can be amplified in individual flies and subsequently disseminated into the mammalian host population. This is compatible with the epidemic population structure of T. brucei in which clonal expansion of a few genotypes in a region occurs against a background of recombination between strains (Oberle et al., 2010). Indeed, beside the natural selection of variants, genetic exchange in T. brucei as well as T. congolense is occurring in the tsetse fly. The cellular mechanisms underlying this sexual stage in the tsetse fly remain to be elucidated. Recently, Gibson's lab demonstrated the existence of intraclonal mating during T. brucei fly transmission, in addition to the previously described interclonal recombination, which makes it unlikely that T. brucei remains genetically unaltered during fly transmission (Peacock et al., 2009). Homologs of meiotic genes were identified in the T. brucei genome and the expression of three functionally distinct meiosis-specific proteins in the nucleus of a specific epimastigote cell type has been demonstrated, defining a previously unidentified developmental trypanosome stage in the tsetse fly salivary glands (Peacock et al., 2011). Expression occurred in clonal and mixed infections, indicating that the meiotic program is an intrinsic but hitherto cryptic part of the developmental cycle of trypanosomes. In experimental crosses, expression of the meiosis-specific proteins was observed to occur before cell fusion (Peacock et al., 2011). However, the actual trypanosome fusion event has not yet been described.

It remains puzzling that the T. brucei parasite developed a complex sexual exchange mechanism in the tsetse salivary glands, knowing that in natural situations the probability of two different $T$. brucei strains successfully developing and meeting in the salivary glands is extremely low. Of course, the fact that even intraclonal mating results in recombination events that introduce genetic variability in the metacyclic trypanosomes could be considered as an evolutionary advantage. The probability of mating events for $T$. congolense in the tsetse fly are likely to be higher, as suggested by recent population genetics analyses where the observed parasite genotypic diversity could only be explained by the occurrence of frequent mating (Morrison et al., 2009). However, no experimental information on where this mating event occurs during development in the tsetse fly is yet available. For T. vivax, nothing is known so far about the existence of mating but the genetic diversity between populations is estimated to be low (Tait et al., 2011). The latter could result from the occurrence of non-cyclical transmission and/or the absence of genetic exchange during cyclical development in tsetse flies.

\section{TSETSE IN A TEST-TUBE?}

Unravelling the intricate interactions between African trypanosomes and the tsetse vector remains a challenge due to technical constraints and time consuming experimental procedures. Recently, by overexpressing a single RNA-binding protein, TbRBP6, in cultured non-infectious T. brucei trypanosomes, Kolev et al. (2012) in vitro reproduced the developmental stages that have been observed in tsetse, including the generation of infective metacyclic forms that express the variant surface glycoprotein. It can be stated that this recent finding is one of the major breakthroughs of the last decades in T. brucei research, opening avenues to experimentally unravel the parasite biology during the final development into the infective metacyclic stage. The developmental cycle of $T$. congolense can be reliably reproduced in vitro and cultures yield large numbers of trypanosomes of different life cycle stages (Coustou et al., 2010). Another advancement was also recently achieved by the optimization and standardization of noninfective T. vivax epimastigote axenic cultures leading to in vitro differentiation into metacyclic infective forms (D'Archivio et al., 2011). However, although these methods could provide an easy access to the intricate molecular and cellular events leading trypanosomes to the acquisition of infectivity, they will not replace the use of the tsetse fly infection model to unravel the complex cross-talks between these two organisms.

\section{SOME REFLECTIONS FOR FUTURE RESEARCH}

For T. brucei, our understanding of the developmental cycle in the fly has been steadily improving by information emerging from recent molecular and cell biological analyses (Sharma et al., 2009; Dyer et al., 2013). T. congolense shares a common migratory pathway with $T$. brucei but the transitional developmental stages in the foregut and mouthparts are remarkably different in these two trypanosome species (Peacock et al., 2012). Our knowledge on T. vivax development in the tsetse vector is currently very limited, but the increasing socio-economic impact of this widespread parasite prompts in-depth re-investigation of its life cycle.

A strikingly common feature of the three developmental programs is the passage through the epimastigote morphotype. Here, details of transitional forms are especially sparse for T. vivax and T. congolense. One of the key questions is whether there is any form equivalent to the asymmetric dividing stage of $T$. brucei. Moreover, the biological reason for this obligatory morphotype switch remains elusive. Is the epimastigote configuration more adapted to cell fixation via the flagellum compared to the trypomastigote? This flagellum attachment to a solid substrate is a prerequisite for multiple parasite transmission as it provides an efficient way to maintain a pool of progenitor cells that continuously produces infective forms without being expelled with the saliva during tsetse fly feeding.

Is the complex and directional development of T. brucei (and T. congolense) in the tsetse driven by an active parasite sensing? Indeed, this journey is highly organized in time and space 
where scanning of the different micro-environments by the parasite can be assumed to be essential for proper cell orientation during migration as well as for initiation of cell cycle switches and differentiation. During this travel through the dark continent, the trypanosome flagellum could act as a sensory organelle, especially through the MAP kinase pathway (Rotureau et al., 2009). Supporting this hypothesis, MAP kinase kinase 1 null mutants of a fly-transmissible strain were able to establish midgut infections at normal rates and intensities, but were incapable of colonizing the salivary glands, suggesting that the signaling cascades involving MAPKK1 are indispensable for transmission of T. brucei (Morand et al., 2012). The role of the T. brucei transmembrane protein PSSA-2 might also be to sense and transmit signals contributing to the parasite's decision to divide, differentiate or migrate (Fragoso et al., 2009). Whether transmembrane signaling molecules such as adenylate cyclases are actively involved in sensing and steering the parasite developmental program in tsetse is still an open question (Salmon et al., 2012).

\section{REFERENCES}

Aksoy, S., Gibson, W. C., and Lehane, M. J. (2003). Interactions between tsetse and trypanosomes with implications for the control of trypanosomiasis. Adv. Parasitol. 53, 1-83. doi: 10.1016/S0065-308X(03)53002-0

Brun, R., Blum, J., Chappuis, F., and Burri, C. (2009). Human African trypanosomiasis. Lancet 375, 148-159. doi: 10.1016/S0140-6736 (09)60829-1

Coustou, V., Guegan, F., Plazolles, N., and Baltz, T. (2010). Complete in vitro life cycle of Trypanosoma congolense: development of genetic tools. PLoS Negl. Trop. Dis. 4:e618. doi: 10.1371/journal.pntd.0000618

D'Archivio, S., Medina, M., Cosson, A., Chamond, N., Rotureau, B., Minoprio, P., et al. (2011). Genetic engineering of Trypanosoma (Dutonella) vivax and in vitro differentiation under axenic conditions. PLoS Negl. Trop. Dis. 5:e1461. doi: 10.1371/journal.pntd.0001461

Delespaux, V., Dinka, H., Masumu, J., Van den Bossche, P., and Geerts, S. (2008). Five-fold increase in Trypanosoma congolense isolates resistant to diminazene aceturate over a seven-year period in Eastern Zambia. Drug Resist. Updat. 11, 205-209. doi: 10.1016/j.drup.2008. 10.002

Dyer, N. A., Rose, C., Ejeh, N. O., and Acosta-Serrano, A. (2013). Flying tryps: survival and maturation of trypanosomes in tsetse flies. Trends Parasitol. 29, 188-196. doi: 10.1016/j.pt.2013.02.003

Fevre, E. M., Picozzi, K., Jannin, J., Welburn, S. C., and Maudlin, I. (2006). Human African trypanosomiasis: epidemiology and control. Adv. Parasitol. 61, 167-221. doi: $\quad 10.1016 / \mathrm{S} 0065-308 \mathrm{X}(05)$ 61005-6

Fragoso, C. M., Schumann Burkard, G., Oberle, M., Renggli, C. K., Hilzinger, K., and Roditi, I. (2009). PSSA-2, a membranespanning phosphoprotein of Trypanosoma brucei, is required for efficient maturation of infection. PLoS ONE 4:e7074. doi: 10.1371/journal.pone.0007074

Hoare, C. A. (1972). The Trypanosomes of Mammals: A Zoological Monograph. Oxford: Blackwell Scientific Publications.

Hoare, C. A., and Wallace, F. G. (1966). Developmental stages of trypanosomatid flagellates: a new terminology. Nature 212, 1385-1386. doi: $10.1038 / 2121385 \mathrm{a} 0$

Jefferies, D., Helfrich, M. P., and Molyneux, D. H. (1987). Cibarial infections of Trypanosoma vivax and T. congolense in Glossina. Parasitol. Res. 73, 289-292. doi: 10.1007/BF00531079

Kolev, N. G., Ramey-Butler, K., Cross, G. A., Ullu, E., and Tschudi, C. (2012). Developmental progression to infectivity in Trypanosoma brucei triggered by an RNA-binding protein. Science 338, 1352-1353. doi: 10.1126/science.1229641

Moloo, S. K., and Gray, M. A. (1989). New observations on cyclical development of Trypanosoma vivax in Glossina. Acta Trop. 46, 167-172. doi: 10.1016/ 0001-706X(89)90033-8

Morand, S., Renggli, C. K., Roditi, I., and Vassella, E. (2012). MAP kinase kinase 1 (MKK1) is essential for transmission of Trypanosoma brucei by Glossina morsitans. Mol.

Recently, an intriguing phenomenon of social motility in African trypanosomes has been documented in in vitro experimental settings (Oberholzer et al., 2010) but the in vivo biological relevance inside the tsetse fly remains to be elucidated.

The recent publications of tsetse and trypanosome genomes as well as the development and refinement of molecular and cellular tools have paved the way for new functional approaches to study the African trypanosomes' development in their vectors. Morphological remodeling, motility, metabolism, control of differentiation and especially sensing are some of the most promising areas to identify targets to block trypanosome development and/or transmission.

\section{ACKNOWLEDGMENTS}

This work was funded by the Institut Pasteur (Brice Rotureau), and by the ERC-Nanosym project, the Research FoundationFlanders, the InterUniversity Attraction Pole program P7/41 and the Institute of Tropical Medicine (Jan Van Den Abbeele).

Biochem. Parasitol. 186, 73-76. doi: 10.1016/j.molbiopara.2012.09.001

Morrison, L. J., Tweedie, A., Black, A., Pinchbeck, G. L., Christley, R. M., Schoenefeld, A., et al. (2009). Discovery of mating in the major African livestock pathogen Trypanosoma congolense. PLoS ONE 4:e5564. doi: 10.1371/journal.pone. 0005564

Oberholzer, M., Lopez, M. A. McLelland, B. T., and Hill, K. L. (2010). Social motility in African trypanosomes. PLoS Pathog. 6:e1000739. doi: 10.1371/journal. ppat.1000739

Oberle, M., Balmer, O., Brun, R., and Roditi, I. (2010). Bottlenecks and the maintenance of minor genotypes during the life cycle of Trypanosoma brucei. PLoS Pathog. 6:e1001023. doi: 10.1371/journal.ppat. 1001023

Peacock, L., Cook, S., Ferris, V., Bailey, M., and Gibson, W. (2012). The life cycle of Trypanosoma (Nannomonas) congolense in the tsetse fly. Parasit. Vectors 5:109. doi: 10.1186/1756-3305-5-109

Peacock, L., Ferris, V., Bailey, M., and Gibson, W. (2007). Dynamics of infection and competition between two strains of Trypanosoma brucei brucei in the tsetse fly observed using fluorescent markers. Kinetoplastid Biol. Dis. 6:4. doi: 10.1186/1475-9292-6-4

Peacock, L., Ferris, V., Bailey, M., and Gibson, W. (2009). Intraclonal mating occurs during tsetse transmission of Trypanosoma brucei. Parasit. Vectors 2, 43. doi: 10.1186/17563305-2-43

Peacock, L., Ferris, V., Sharma, R., Sunter, J., Bailey, M., Carrington,
M., et al. (2011). Identification of the meiotic life cycle stage of Trypanosoma brucei in the tsetse fly. Proc. Natl. Acad. Sci. U.S.A. 108, 3671-3676. doi: 10.1073/pnas. 1019423108

Roditi, I., and Lehane, M. J. (2008) Interactions between trypanosomes and tsetse flies. Curr. Opin. Microbiol. 11, 345-351. doi: 10.1016/j.mib.2008.06.006

Rotureau, B., Morales, M. A., Bastin, P., and Spath, G. F. (2009). The flagellum-MAP kinase connection in Trypanosomatids: a key sensory role in parasite signaling and development? Cell. Microbiol. 11, 710-718. doi: 10.1111/j.1462-5822. 2009.01295.x

Rotureau, B., Subota, I., and Bastin, P. (2011). Molecular bases of cytoskeleton plasticity during the Trypanosoma brucei parasite cycle. Cell. Microbiol. 13, 705-716. doi: 10.1111/j.1462-5822.2010.01566.x

Rotureau, B., Subota, I., Buisson, J., and Bastin, P. (2012). A new asymmetric division contributes to the continuous production of infective trypanosomes in the tsetse fly. Development 139, 1842-1850. doi: $10.1242 /$ dev. 072611

Salmon, D., Vanwalleghem, G., Morias, Y., Denoeud, J., Krumbholz, C., Lhomme, F., et al. (2012). Adenylate cyclases of Trypanosoma brucei inhibit the innate immune response of the host. Science 337, 463-466. doi: 10.1126/science. 1222753

Sharma, R., Gluenz, E., Peacock, L., Gibson, W., Gull, K., and Carrington, M. (2009). The heart of darkness: growth and form of Trypanosoma brucei in the tsetse fly. 
Trends Parasitol. 25, 517-524. doi: 10.1016/j.pt.2009.08.001

Sharma, R., Peacock, L., Gluenz, E., Gull, K., Gibson, W., and Carrington, M. (2008). Asymmetric cell division as a route to reduction in cell length and change in cell morphology in trypanosomes. Protist 159, 137-151. doi: 10.1016/j.protis.2007.07.004

Simarro, P. P., Cecchi, G., Franco, J. R., Paone, M., Diarra, A., Ruiz-Postigo, J. A., et al. (2012). Estimating and mapping the population at risk of sleeping sickness. PLoS Negl. Trop. Dis. 6:e1859. doi: 10.1371/journal.pntd.0001859

Simarro, P. P., Cecchi, G., Paone, M., Franco, J. R., Diarra, A., Ruiz, J. A., et al. (2010). The Atlas of human African trypanosomiasis: a contribution to global mapping of neglected tropical diseases. Int. J. Health Geogr. 9:57. doi: 10.1186/1476-072X-9-57

Simarro, P. P., Jannin, J., and Cattand, P. (2008). Eliminating human African trypanosomiasis: where do we stand and what comes next? PLoS Med. 5:e55. doi: 10.1371/journal.pmed.0050055

Subota, I., Rotureau, B., Blisnick, T., Ngwabyt, S., Durand-Dubief, M.,
Engstler, M., et al. (2011). ALBA proteins are stage regulated during trypanosome development in the tsetse fly and participate in differentiation. Mol. Biol. Cell 22, 4205-4219. doi: 10.1091/mbc.E1106-0511

Tait, A., Morrison, L. J., Duffy, C. W., Cooper, A., Turner, C. M., and Macleod, A. (2011). Trypanosome genetics: populations, phenotypes and diversity. Vet. Parasitol. 181, 61-68. doi: 10.1016/j.vetpar.2011.04.024

Tetley, L., and Vickerman, K. (1985). Differentiation in Trypanosoma bru$c e i$ host-parasite cell junctions and their persistence during acquisition of the variable antigen coat. J. Cell. Sci. 74, 1-19.

Van Den Abbeele, J., Caljon, G. De Ridder, K., De Baetselier, P., and Coosemans, M. (2010). Trypanosoma brucei modifies the tsetse salivary composition, altering the fly feeding behavior that favors parasite transmission. PLoS Pathog. 6:e1000926. doi: 10.1371/journal.ppat.1000926

Van Den Abbeele, J., Claes, Y., van Bockstaele, D., Le Ray, D. and Coosemans, M. (1999). Trypanosoma brucei spp. development in the tsetse fly: characterization of the post-mesocyclic stages in the foregut and proboscis. Parasitology 118, 469-478. doi: 10.1017/S0031182099004217

Walshe, D. P., Ooi, C. P., Lehane, M. J., and Haines, L. R. (2009). The enemy within: interactions between tsetse, trypanosomes and symbionts. $A d v$. Insect Physiol. 37, 119-175. doi 10.1016/S0065-2806(09)37003-4

Weiss, B. L., Maltz, M. A., and Aksoy, S. (2012). Obligate symbionts activate immune system development in the tsetse fly. J. Immunol. 188: 3395-3403. doi 10.4049/jimmunol.1103691

Weiss, B. L., Wang, J., Maltz, M. A., Wu, Y., and Aksoy, S. (2013). Trypanosome infection establishment in the tsetse fly gut is influenced by microbiomeregulated host immune barriers. PLoS Pathog. 9:e1003318. doi 10.1371/journal.ppat. 1003318

WHO. (2012). "Research priorities for Chagas disease, human African trypanosomiasis and leishmaniasis," in WHO Technical Report Series, 1st Edn. ed WHO (Geneva: WHO).

Conflict of Interest Statement: The authors declare that the research was conducted in the absence of any commercial or financial relationships that could be construed as a potential conflict of interest.

Received: 24 July 2013; paper pending published: 20 August 2013; accepted: 29 August 2013; published online: 18 September 2013.

Citation: Rotureau $B$ and Van Den Abbeele J (2013) Through the dark continent: African trypanosome development in the tsetse fly. Front. Cell. Infect. Microbiol. 3:53. doi: 10.3389/ fcimb.2013.00053

This article was submitted to the journal Frontiers in Cellular and Infection Microbiology.

Copyright (c) 2013 Rotureau and Van Den Abbeele. This is an open-access article distributed under the terms of the Creative Commons Attribution License (CC BY). The use, distribution or reproduction in other forums is permitted, provided the original author(s) or licensor are credited and that the original publication in this journal is cited, in accordance with accepted academic practice. No use, distribution or reproduction is permitted which does not comply with these terms. 